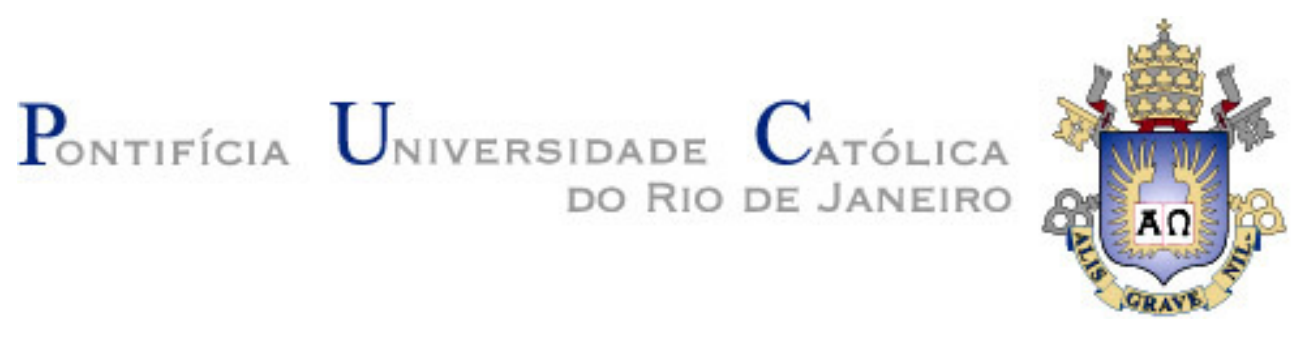

Mariana Luísa Garcia Braido

O desenvolvimento afetivo de bebês com

risco de autismo

Tese de Doutorado

Tese apresentada ao Programa de PósGraduação em Psicologia Clínica da PUC-Rio como requisito parcial para obtenção do grau de doutor em Psicologia

Orientadora: Carolina Lampreia

Rio de Janeiro

Julho de 2011 
Mariana Luísa Garcia Braido

\title{
O desenvolvimento afetivo de bebês com risco de autismo
}

Tese apresentada como requisito parcial para obtenção do grau de Doutor pelo Programa de Pós-Graduação em Psicologia Clínica do Departamento de Psicologia do Centro de Teologia e Ciências Humanas da PUC-Rio. Aprovada pela Comissão Examinadora abaixo assinada

\author{
Prof ${ }^{a}$. Carolina Lampreia \\ Orientadora \\ Departamento de Psicologia - PUC-Rio \\ Profa. Denise Streit Morsch \\ Departamento de Psicologia - PUC-Rio \\ Prof ${ }^{a}$. Silvia Maria AbuJamra Zornig \\ Departamento de Psicologia - PUC-Rio \\ Profa. Cláudia Maria Passos Ferreira \\ IMS/UERJ \\ Prof ${ }^{a}$. Maria da Conceição Diniz Pereira de Lyra \\ Departamento de Psicologia - UFPE \\ Profá. Denise Berruezo Portinari \\ Coordenadora Setorial de Pós-Graduação e \\ Pesquisa do Centro de Teologia e Ciências \\ Humanas - PUC-Rio
}


Todos os direitos reservados. É proibida a reprodução total ou parcial do trabalho sem autorização da universidade, da autora e da orientadora.

Mariana Luísa Garcia Braido

Graduada em Psicologia pela Universidade Federal de São Carlos (UFSCar), desenvolveu trabalho de atendimento clínico individualizado de crianças com autismo e outros transtornos do desenvolvimento antes de seu ingresso na pós-graduação. Concluiu o mestrado em Psicologia Clínica pela Pontifícia Universidade Católica do Rio de Janeiro investigando o desenvolvimento típico e atípico de bebês em suas interações sócio-afetivas.

Ficha Catalográfica

Braido, Mariana Luísa Garcia

O desenvolvimento afetivo de bebês com risco de autismo / Mariana Luísa Garcia Braido ; orientadora: Carolina Lampreia. - 2011. 143 f. ; $30 \mathrm{~cm}$

Tese (doutorado)-Pontifícia Universidade Católica do Rio de Janeiro, Departamento de Psicologia, 2011.

Inclui bibliografia

1. Psicologia - Teses. 2. Autismo. 3. Identificação precoce. 4. Bebês de risco. 5. Desenvolvimento afetivo. 6. Interação adultobebê. I. Lampreia, Carolina. II. Pontifícia Universidade Católica do Rio de Janeiro. Departamento de Psicologia. III. Título. 
Para as famílias que participaram deste estudo e me ensinaram tanto. 


\section{Agradecimentos}

À Carolina Lampreia por estes seis anos de dedicação ao meu aprimoramento acadêmico, serei eternamente grata.

À Roberta Caminha pela amizade e companhia durante a coletada de dados, pelo entusiasmo com que olha para o meu trabalho e interagiu com os bebês nas filmagens, minha profunda gratidão.

As famílias que participaram deste estudo, especialmente às mães, com seus estilos particulares de interagir com seus bebês, ensinaram-me imensamente.

À Denise Morsch que me ofereceu a oportunidade de observar seu trabalho de avaliação de bebês e pela participação na avaliação dos bebês deste estudo.

À Carla Gikovate pela generosa participação na avaliação dos bebês deste estudo.

À Olivia Balster Fiore Correia, Ana Maria Camelo Campos e Stephan Malta Oliveira, colegas de estudos e profissionais empenhados e dedicados a compreender o autismo, cujas posições e idéias fizeram com que eu arejasse as minhas.

Aos professores da PUC-Rio, em especial à Flávia Sollero, sempre entusiasta e interessada pelo meu trabalho.

Aos funcionários do Departamento de Psicologia, em especial Marcelina e Vera, que cuidam das burocracias da nossa vida na instituição para que possamos trabalhar com tranqüilidade.

As minhas colegas de mestrado e doutorado que eu conheci na PUC-Rio e levei para a vida como amigas: Daniela Vergueiro Rodrigues, Carolina Guimarães e Silva, Carolina Campos Borges, Emmy Uehara.

Aos alunos de iniciação científica Ana Luisa Dau, Karin Müller, Gilberto Desidério e Vicente Carneiro.

Ao meu marido, Luis Henrique Bertolino Braido, que sempre me incentivou e valorizou o meu trabalho.

Aos meus amigos e familiares que puderam desculpar minha ausência durante os anos de mestrado e doutorado.

A CAPES, FAPERJ e PUC-Rio, pelos auxílios concedidos, sem os quais este trabalho não poderia ter sido realizado. 


\section{Resumo}

Braido, Mariana Luísa Garcia; Lampreia, Carolina (orientadora). O desenvolvimento afetivo de bebês com risco de autismo. Rio de Janeiro, 2011. 143p. Tese de Doutorado - Departamento de Psicologia, Pontifícia Universidade Católica do Rio de Janeiro.

O reconhecimento de sinais precoces do autismo é crucial para o encaminhamento de crianças à intervenção precoce. Um corpo de conhecimento consistente de sinais de risco no segundo ano de vida foi estabelecido a partir de estudos retrospectivos de vídeos familiares. A identificação de sinais de risco no primeiro ano de vida passou a ser um desafio na área de autismo. A metodologia utilizada na busca de tais sinais é de acompanhamento prospectivo de bebês de risco, irmãos de crianças com autismo, por terem chances aumentadas de diagnóstico. O presente estudo prospectivo de casos múltiplos teve o objetivo de acompanhar o desenvolvimento de dois bebês de risco entre 3 e 12 meses, compará-lo ao de dois bebês sem histórico familiar de autismo, e identificar diferenças no desenvolvimento deles que pudessem sinalizar risco de autismo. Estes bebês foram filmados mensalmente em interações sociais com um adulto que foram analisadas de acordo com categorias afetivas, interativas e manejo. Narrativas históricas do desenvolvimento deles foram elaboradas. Os resultados da análise das categorias não mostraram diferenças entre os bebês. Entretanto, as narrativas históricas mostraram que, entre 8 e 12 meses, um dos bebês de risco teve dificuldade de igualar a intensidade de seu afeto com a intensidade do afeto do adulto e de rastrear a face do adulto durante jogo de esconder a face. Aos 21 meses este bebê foi encaminhado preventivamente para intervenção precoce. $\mathrm{O}$ uso de categorias afetivas e análise qualitativa da equiparação do afeto do bebê com o do adulto na avaliação de bebês de risco e em protocolos de rastreamento de autismo é discutido.

\section{Palavras-chave}

Autismo; identificação precoce; bebês de risco; desenvolvimento afetivo; interação bebê-adulto. 


\section{Abstract}

Braido, Mariana Luísa Garcia; Lampreia, Carolina (advisor). Affective development in infants at risk for autism. Rio de Janeiro, 2011. 143p. D.Sc. Thesis - Departamento de Psicologia, Pontifícia Universidade Católica do Rio de Janeiro.

Recognition of early signs of autism is crucial to early intervention. Signs of autism in the second year of life have already been established through retrospective home video studies of children diagnosed with autism. On the aim of having infants reaching intervention earlier than two years old, research has been conducted with a promising methodology. It consisted of following prospectively development of high risk infants. Since autism diagnosis is more frequent in younger siblings of children with autism, they are considered to be at increased risk for the disorder. On the other hand, infants without family history of autism are considered to be at low risk for autism. In the present prospective multiple case study, development of two high risk infants were followed during the first year and compared to development of two low risk infants. Interactions of these infants with an adult were recorded in video monthly. These videos were analyzed according to affective, interactive and coping categories. Historical narratives of interactions from 3 to 12 months were conducted as well. Results of categories analysis did not showed differences among infants of both groups. Interestingly, historical narratives showed that, between 8 and 12 month, one of the high risk infants had difficulties in matching his affect intensity with affect intensity of his interactive partner and also on tracking adults face during peek-a-boo game. At 21 months this infant was preventively referred to early intervention. Implications of using affective categories and affective matching in assessing high risk infants as well as in screening instruments are discussed.

\section{Keywords}

Autism; early signs; high risk siblings; affective development; infant-adult interaction. 


\section{Sumário}

1. Introdução

2. O desenvolvimento afetivo no primeiro ano de vida e suas

implicações para o estudo de bebês com risco de autismo

2.1. Uma nota sobre os termos afeto e emoção 19

2.2. O repertório do bebê e sua capacidade de engajamento afetivo 20

2.3. O repertório do adulto 23

2.4. As interações diádicas ou comunicação afetiva de bebês com desenvolvimento típico $\quad 25$

2.5. Padrões de interações atípicas e o caso do autismo 31

2.6. Subsídios para a investigação de interações adulto-bebê

com risco de autismo 34

3. Os estudos prospectivos e retrospectivos de sinais $\begin{array}{ll}\text { precoces de autismo } & 37\end{array}$

3.1. Estudos retrospectivos de vídeos familiares 38

3.2. Estudos prospectivos com amostras de risco 44

3.3. Conclusão 52

4. Analisando interações mãe-bebê com risco e sem risco de autismo 56

4.1. A escolha metodológica $\quad 57$

4.2. Método $\quad 58$

4.3. Resultados 68

4.4. Discussão 118

$\begin{array}{ll}\text { 5. Considerações finais } & 121\end{array}$

5. 1. Limitações do estudo e direções futuras 126

6. Referências Bibliográficas 129

$\begin{array}{ll}\text { 7. Anexos } & 138\end{array}$

$\begin{array}{ll}\text { 7.1. Anexo } 1 & 138\end{array}$

$\begin{array}{ll}\text { 7. 2. Anexo } 2 & 143\end{array}$ 
Quatro décadas atrás o interesse científico começou a enfocar uma nova teoria sobre como vontade e emoção humanas são imediatamente compartilháveis com os outros pelos gestos do corpo e pela voz. Muitos pesquisadores que contribuíram com este novo jeito de olhar para a natureza humana, (...), fazendo observações independentes de mães e bebês em comunicação natural e mutuamente prazerosa, consideraram que a vitalidade dos gestos comunicativos é suficiente para a criação de histórias memoráveis.

Stephen Malloch e Colwyn Trevarthen, Communicative Musicality, p. 1, 2009. [tradução minha] 